\title{
Unexpected Honeycomb-like Structural Analogues Incorporating Longer Bipyridyl Linkers
}

\author{
Seong-Hyeon Park ${ }^{1}$, Hyun-Sung Jang ${ }^{1}$, Woo-Hyeong Kang ${ }^{1}$ and Eun-Young Choi ${ }^{* 1,2}$ \\ ${ }^{1}$ Korea Science Academy of KAIST, Korea \\ ${ }^{2}$ Korea Advanced Institute of Science and Technology (KAIST), Korea
}

*Corresponding author: Eun-Young Choi, Korea Science Academy of KAIST, Korea Advanced Institute of Science and Technology (KAIST), Korea

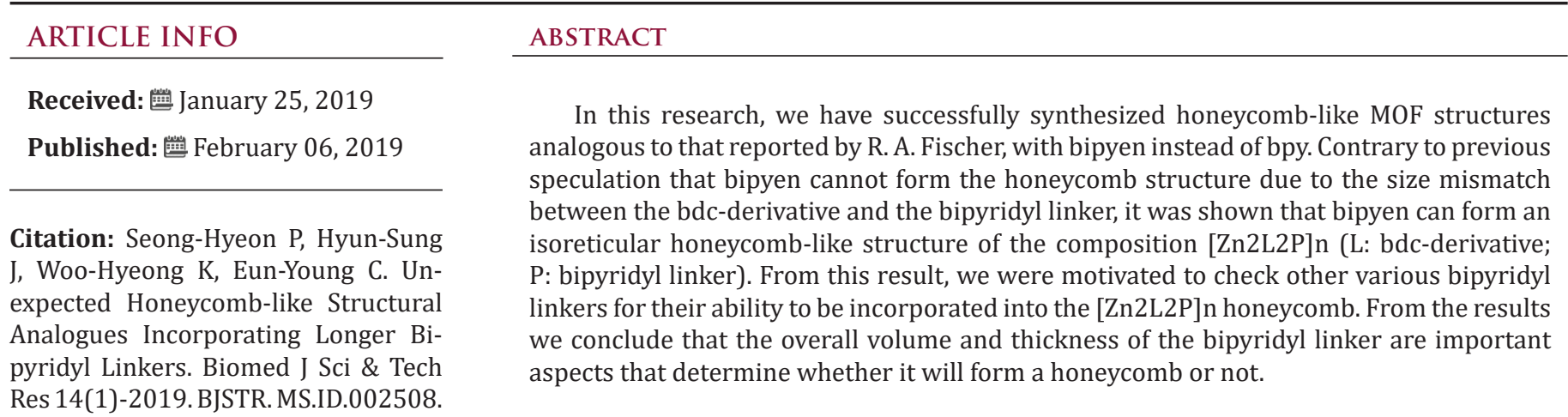

\section{Introduction}

Metal-Organic Frameworks (MOFs) present remarkable customizability of chemical and physical properties depending on its highly variable compositions. Its versatile micropore environment hints various potential applications of MOFs such as gas storage [1], gas separation [2], heterogeneous catalyst [3], and specific sensor [4]. It is generally synthesized from coordination between metal cation and organic ligands to create diverse compositions and so as structures and chemical environments of crystal pores. Therefore, making use of various linkers usually gives rise to unexpected structural changes or analogues of certain structure, which greatly spotlights the importance of researching and understanding how the system reacts to changes. Henke et al. [5] had previously reported the discovery of honeycomblike framework with composition [Zn2L2P]n (L = 2,5-bis(2methoxyethoxy)-1,4-benzenedicarboxylate; $\mathrm{P}=4,4$ '-bipyridine, bpy). The structure has unidirectional channels with substituted side chains from 1,4-benzenedicarboxylate (bdc) linker directed inside, inducing exceptionally high selective adsorption property toward carbon dioxide gas over nitrogen gas [5] The framework exhibited usefulness in commercial usage such as carbon dioxide separation based on its particular adsorption property if its water stable analogue could be found. It also hinted the way to derive unexpected MOF topology from the [Zn2L2P]n system.

From previous works [6], the prerequisites for the formation of the honeycomb-like structure from the [Zn2L2P]n system is thought to be the following: first, the size of the $\mathrm{L}$ (bdc-derivative) and $\mathrm{P}$ (bipyridyl linker) must match each other, and second, there must be sufficient steric hindrance such that the pillared paddlewheel structure will be thermodynamically unfavorable. In this research, we aimed to precisely describe the boundaries of the required conditions by changing the two conditions - the length of the bipyridyl linker and the steric hindrance between the linkers.

\section{Results \& Discussion}

Following the naming system of EY Choi et al. [7] the bdcderivative ligand was dubbed $\mathrm{Cn}$-acid, with $\mathrm{n}$ being the number of carbons in the substituents. Other ligand molecules used in this study is summarized in Figure 1 Cn-acids ( $n=5 \sim 10,12,14$ ) were selected to be the bdc-derivative linker of choice because of its highly variable side chain length. Stoichiometric amounts of 
zinc nitrate hexahydrate, $\mathrm{Cn}$-acid and bipyen were dissolved in diethylformamide (DEF), and heated to $100^{\circ} \mathrm{C}$ for 120 hours to yield isoreticular honeycomb-like framework. As Figure 2 indicates, PXRD patterns of our new crystal synthesized with Cn-acid $(5 \leq n \leq 7)$ shows great coincidence with theoretical PXRD pattern of honeycomb-like structure. Moreover, the synthesized structure showed a similar thermal stability profile with that of the bpy- analogue with the same Cn-acid, as confirmed by TGA as shown in Figure 3. Following the successful synthesis of an analogous honeycomb-like structure with bipyen instead of bpy, we were motivated to test other bipyridyl linkers, like bpe and dabco. As shown in Figures 4-6, using dabco and bpe led to different results. Significantly shorter dabco than bpy lead to paddle-wheel structure ( $n=6$ ) or layered polymers ( $n \geqq 10$ ) as shown in Figure 6 . (a)

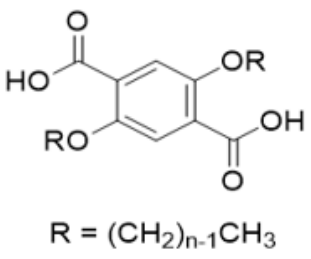

(c)<smiles>C(=C/c1ccncc1)\c1ccncc1</smiles>

(E)-1,2-di(pyridin-4-yl)ethene (b)

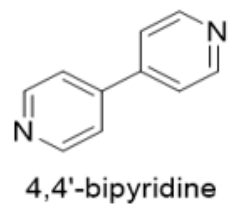

(d)<smiles>c1cc(CCc2ccncc2)ccn1</smiles>

1,2-di(pyridin-4-yl)ethane

(e)<smiles>C1CN2CCN1CC2</smiles>

1,4-diazabicyclo[2.2.2]octane

Figure 1: Structure of ligands: (a) Cn-acids $=2,5$-bis(alkyloxy) terephthalic acid (b) bpy $=4,4^{\prime}$-bipyridyl (c) bipyen=(E)-1,2di(pyridin-4-yl)ethene $(\mathrm{d})$ bpe = 1,2-di(pyridin-4-yl)ethane (e) dabco = 1,4-diazabicyclo[2.2.2] octane.

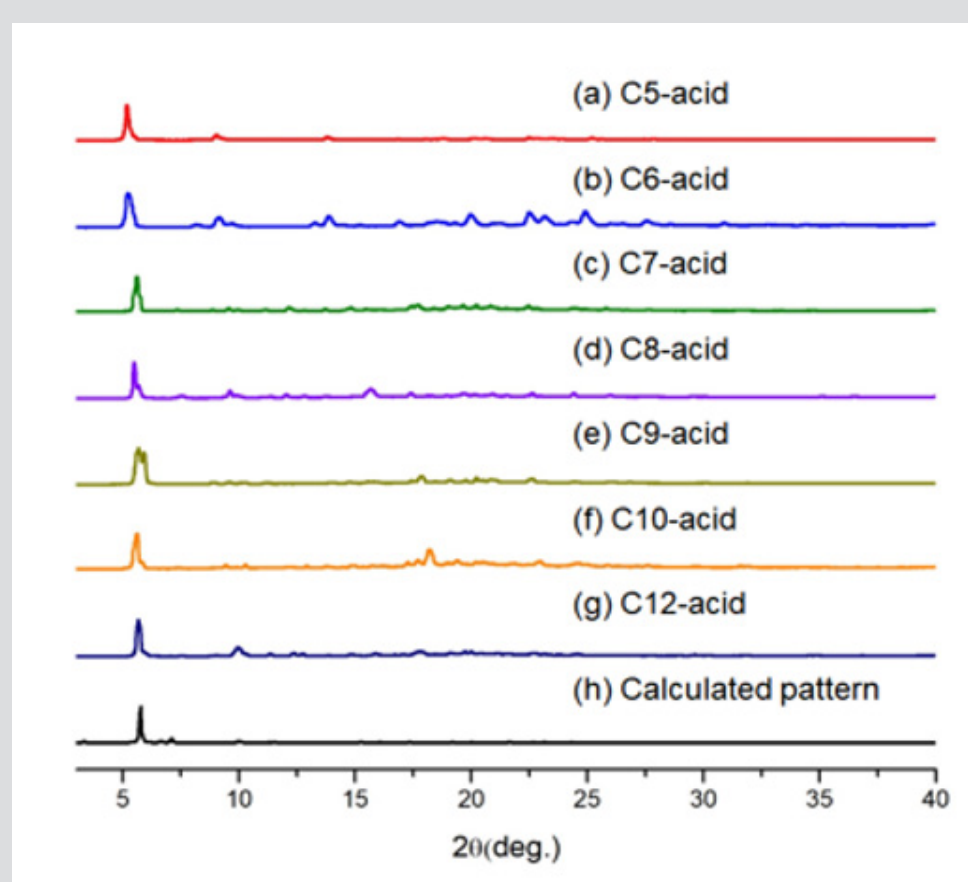

Figure 2: Powder X-ray Diffraction Patterns of $\mathrm{Zn} / \mathrm{Cn}$-acid(n=5-10, 12)/bipyen samples. (h) Calculated pattern is obtained from single crystal structure of honeycomb structure in CSD [9] (reference code: EVOTIT). 
(a) C6-acid and bpy

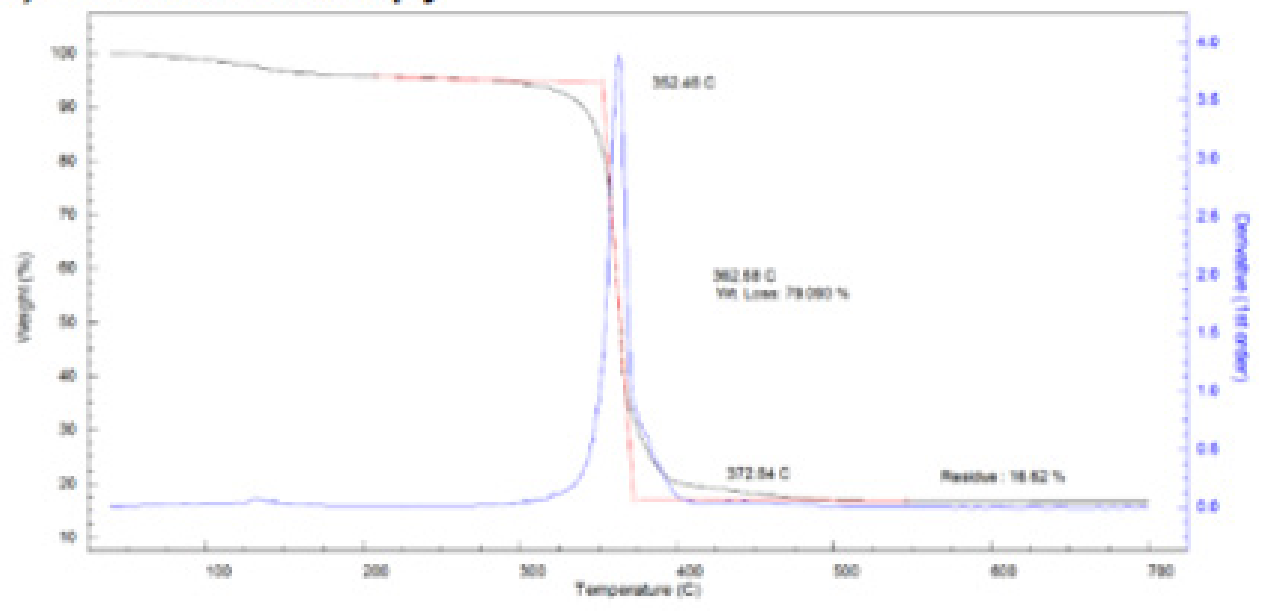

(b) C6-acid and bipyen

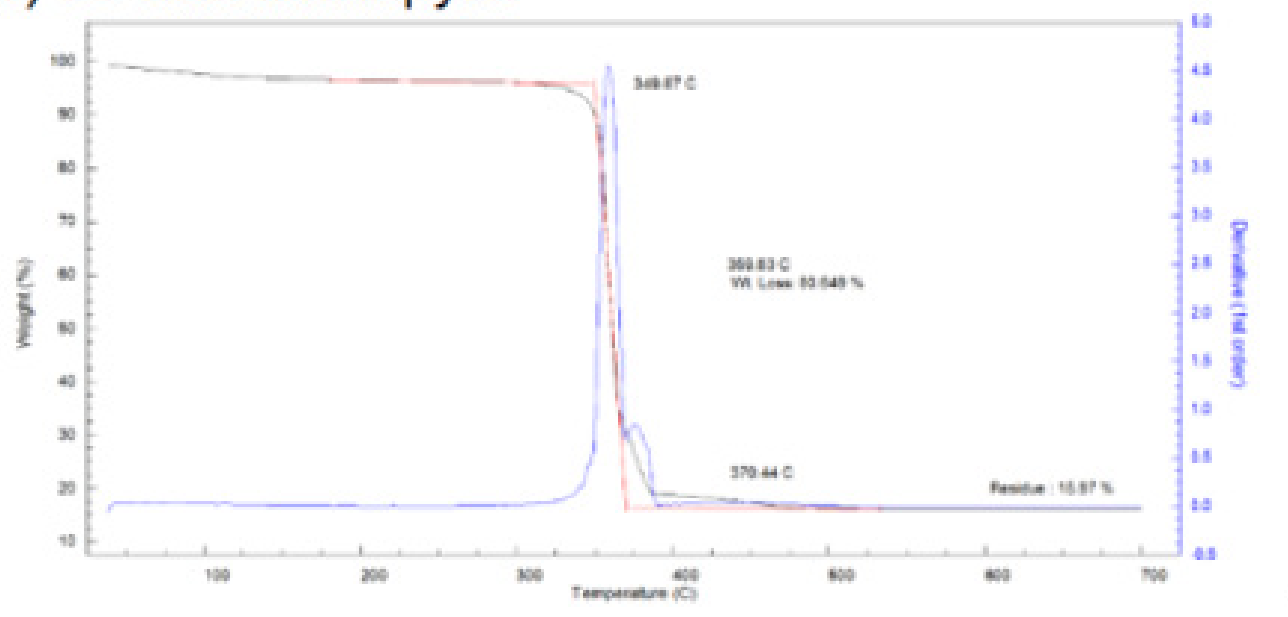

Figure 3: TGA analysis of (a) Zn/C6-acid/bpy (b) Zn/C6-acid/bipyen.

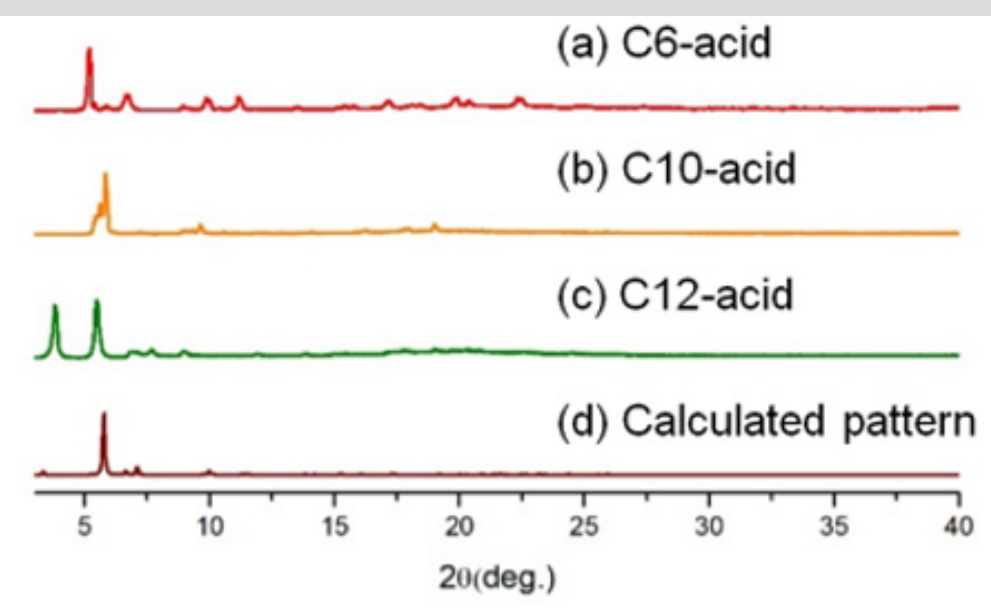

Figure 4: Powder X-Ray Diffraction patterns of Zn/Cn-acid(n=6, 10, 12)/bpe. 


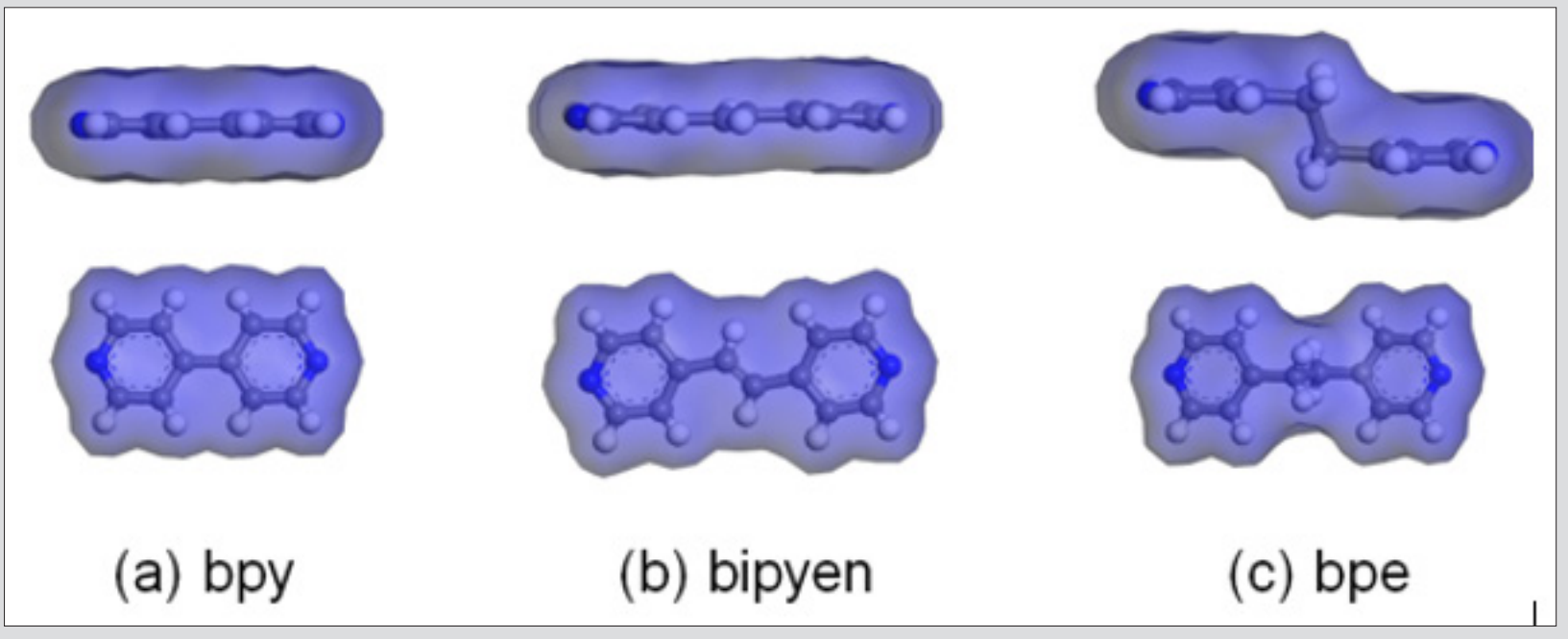

Figure 5: Connolly surface of ligands (bpy, bipyen, and bpe) those are detached from the reported structures in the CSD.[9] (reference codes: LOHYOY(a), COPWAH(b), BOYEK(c)).

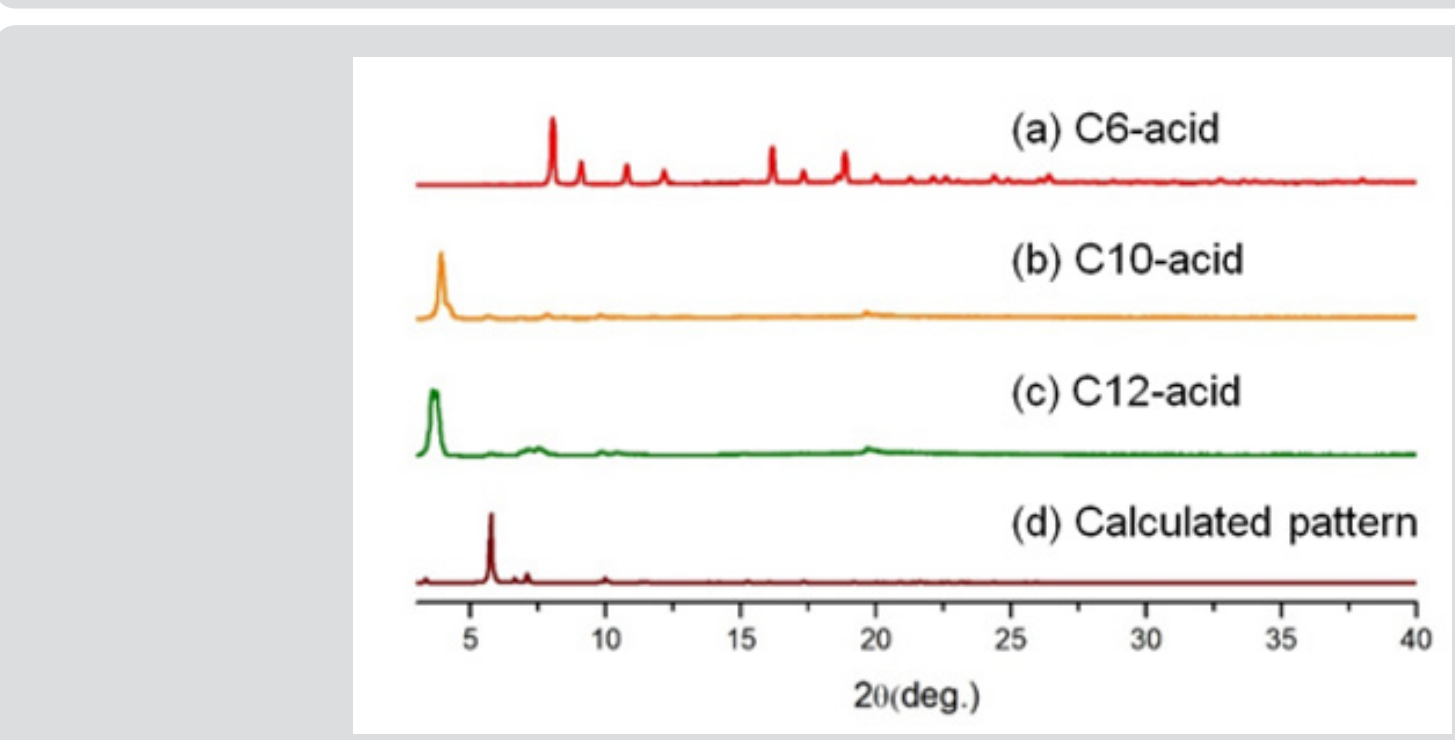

Figure 6: Powder X-Ray Diffraction patterns of $\mathrm{Zn} / \mathrm{Cn}-\operatorname{acid}(\mathrm{n}=6,10,12)$ / dabco.

Even though bpe has a very similar structure to that of bipyen, differing by only the number of bonds between the two pyridinyl groups, bpe does not form frameworks analogous to that of the original honeycomb structure for large $n$. We attribute this to the structural property of the honeycomb structure. In the original structure synthesized from bpy, BME-bdc(2,5-bis(methoxyethoxy)-bdc), and Zn, the "walls" of the honeycomb are composed of alternating ligands - bpy and BME-bdc. The space between the layers do not provide enough volume to incorporate bpe, which is substantially "thicker" than bipyen. This leads to structural instability, which leads to the formation of other structures when the excessive steric hindrance caused by long side chains start to undermine the thermodynamic stability of the honeycomb-like structure in case of $n \geq 12$. The difference between bpe and bipyen is well illustrated in Figure 5. While it has been previously reported that the similarity of size between the bipyridyl linker and the bdc-derivative linker is a prerequisite of formation of the honeycomb-like structure in the [Zn2L2P] system8, our results indicate otherwise [9].

We claim that other bipyridyl linkers with differing sizes can also form the honeycomb-like structure, unless the size mismatch is extremely large. In case of dabco, which is substantially smaller than the bdc linker, the honeycomb-like structure does not form, with the pillared paddlewheel structure forming instead, as shown in Figure 6.

\section{Conclusion}

In conclusion, we have synthesized a previously unreported honeycomb-like structure, with bipyen instead of bpy. In previous works it is suggested that the bdc derivative linkers and the bipyridyl linkers must have similar lengths in order to form the 
unique honeycomb-like structure with one-directional channels $[5,8]$ However, the overall dimensions of the bipyridyl linker seems to be the more important factor for the determination of structure. More specifically, we suggest that there are three important parameters from the overall dimensions of the ligand molecule to be considered; length, thickness, and side group volume.

We assessed the outcomes of changing each parameter in MOF synthesis. First, the length of the bipyridyl linkers were varied, by using dabco, bpy, bipyen, and bpe. From this, we concluded that there was indeed a certain range of suitable lengths, but it was not as strict and narrow, as it was known to be before. The fact that bipyen, which is comparably longer that bpy, is successfully incorporated into the honeycomb-like analogous framework, but not bpe in case of long side chains $(n \geq 12)$, even though bpe is actually more suitable length-wise, suggests that the second factor is more important in this particular framework. We attribute this to the fact that the interlayer distance of the 1D channel is substantially shorter than that of other frameworks.

\section{Acknowledgment}

This work was supported by the Korea Science Academy of KAIST with funds from the Ministry of Science and ICT

ISSN: 2574-1241

DOI: 10.26717.BJSTR.2019.14.002508

Eun-Young Choi.Biomed J Sci \& Tech Res

CC (i) This work is licensed under Creative

Submission Link: https://biomedres.us/submit-manuscript.php

\section{References}

1. AR Millward, OM Yaghi (2005) Metal-Organic Frameworks with Exceptionally High Capacity for Storage of Carbon Dioxide at Room Temperature. J Am Chem Soc 127(51): 17998-17999.

2. JR Li, RJ Kuppler, HC Zhou (2009) Selective gas adsorption and separation in metal-organic frameworks. Chem Soc Rev 38(5): 1477-1504.

3. JY Lee, OK Farha, J Roberts, KA Scheidt, Son Binh, et al. (2009) Metalorganic framework materials as catalysts. Chem Soc Rev (5): 1450-1459.

4. FY Yi, D Chen, M Wu, L Han, HL Jiang, et al. (2016) Chemical Sensors Based on Metal-Organic Frameworks. Chem Plus Chem 81(8): 675-690.

5. S Henke, RA Fisher (2011) Gated Channels in a Honeycomb-like ZincDicarboxylate-Bipyridine Framework with Flexible Alkyl Ether Side Chains. J Am Chem Soc 133(7): 2064-2067.

6. S Henke, A Schneemann, S Kapoor, R Winter, RA Fisher, et al. (2012) Zinc-1,4-benzenedicarboxylate-bipyridine frameworks - linker functionalization impacts network topology during solvothermal synthesis. J Mater Chem 22: 909-918.

7. EY Choi, C Gao, HJ Lee, OP Kwon, SH Lee, et al. (2009) Transformation of framework solids into processable metallo-polymers. Chem Commun 48: 7563-7565.

8. Z Zhang, HTH Nguyen, SA Miller, AM Ploskonka, JB DeCoste, et al. (2016) Polymer-Metal-Organic Frameworks (polyMOFs) as Water Tolerant Materials for Selective Carbon Dioxide Separations. J Am Chem Soc 138(3): 920-925.

9. (2018) The drawing was carried out using Material Studio Software.

\begin{tabular}{ll} 
BIOMEDICAL & Assets of Publishing with us \\
RESEARCHES & - Global archiving of articles \\
\hline ISSN: $2574-1241$ & - Immediate, unrestricted online access \\
\hline
\end{tabular}

